\title{
El desarrollo de la artesania y su formalización empresarial
}

Elsie Bonilla de Céspedes

Ingeniera industrial con maestría en Gestión de la Producción y estudios de especialización en el área de calidad y productividad en el Instituto Mexicano de Calidad (IMECCA). Consultora Onudi-Confiep. Auditora de Calidad con certificado SGS-Yarsley International Certification Services Ltd. Profesora de la Universidad de Lima

María Carbajal Pantoja

Bachiller en ingeniería industrial por la Universidad de Lima. Profesora de la misma Universidad

Hoy en día, la artesanía está situada como una de las grandes actividades comerciales exportadoras a escala mundial, por lo cual nuestro país podría comenzar a activar este sector de una forma empresarial, teniendo como objetivos fundamentales la mejora en la calidad, costo y variedad, con el fin de conseguir una mayor competitividad con respecto a otros países.

Este artículo es un avance del proyecto de investigación sobre el tema, el cual busca plantear las políticas necesarias para posibilitar la formación y acumulación de capital, respaldándose en sugerencias para el desarrollo técnico y administrativo de este sector.

En el presente trabajo se pueden observar los antecedentes referidos a la artesanía en el Perú, desde los primeros pobladores hasta su actual situación. Se resaltan las principales características, tales como la manera espontánea y empírica de su desarrollo, su bajo monto de inversión, la tecnología tradicional aplicada y el predominio de un manejo manual en las operaciones. 


\section{Presentación}

Una de las actividades comerciales más dinámicas, a escala mundial, es la exportación de artesanías. Según cifras recientes, los tres primeros lugares en venta internacional de productos artesanales fueron ocupados por la India*, con US\$1.005 millones; Irán, con US $\$ 1.000$ millones; y, México, con US\$600 millones. El Perú exportó alrededor de US\$18 millones; paralelamente se estima que la actividad artesanal da ocupación a más de tres millones de personas, distribuidas en más de 23 mil talleres artesanales registrados formalmente, estimándose que una cifra similar, cuando menos, representa el número de talleres informales.

Reflexionando en torno a las causas que generan la brecha de exportaciones artesanales entre México y Perú -países del mismo continente- llegamos a la conclusión de que más allá de las diferencias basadas en la cantidad de turistas que visitan cada una de las naciones, la artesanía mexicana supera a la nuestra en calidad, costo y variedad.

La escasa calidad en el diseño es una de las mayores debilidades de la producción artesanal peruana de exportación, por cuanto no se basa en los requerimientos y exigencias del mercado internacional actual, que busca productos utilitarios, variados y de estilo moderno. De otro lado, el costo es una función de la eficiencia con que se administra la producción, principalmente, y de manera complementaria los procesos de abastecimiento y venta.

Frente a la necesidad social y económica de fortalecimiento que requiere el sector artesanal peruano, el ingeniero industrial, en particular, tiene la gran oportunidad de impulsar su desarrollo. Algunas de las tareas que pueden involucrar a este profesional son: desarrollo del producto, optimización de procesos, desarrollo de canales de comercialización, entre otros. En ese sentido, la Facultad de Ingeniería Industrial de la Universidad de Lima, viene desarrollando -a través del Centro de Investigación de la Producción Industrial (Cipi) - una serie de acti-

* Datos del presidente del Instituto Peruano de Artesanía (Impar) en el foro "La Artesanía Peruana: Estrategias para su Desarrollo" realizado el 24 de setiembre de 1999. 
vidades encaminadas a contribuir con el desarrollo del sector artesanal peruano como, por ejemplo, el apoyo que brinda a esta investigación y la realización de foros como "La Artesanía Peruana, Estrategias para su Desarrollo", efectuado en setiembre de 1999.

En el presente artículo, que constituye un avance del proyecto de investigación antes mencionado, se tratarán los antecedentes, objetivos, hipótesis a comprobar y metodología general; se añade, además, información sobre las principales líneas artesanales y la proyección de la demanda efectuada por los investigadores.

\section{Antecedentes}

El Perú tiene una cultura tradicional en artesanía, reflejada en hermosas manifestaciones que se muestran a través de los productos pertenecientes a diversas líneas, como cerámica, textilería, peletería, imaginería, arpillería, mates burilados, retablos, etc. La artesanía peruana constituye un sector muy particular dentro de la pequeña o la microempresa, ya que en él existen subsectores productivos muy diferenciados, cada uno de ellos con una problemática específica. Sin embargo, el rubro artesanal ha sido tratado como un todo, sin pensar en todas las diferencias que se pueden presentar en él.

El sector artesanal cuenta con una gran variedad de talleres dedicados a diferentes líneas de producción a lo largo del territorio nacional, pero no hay un estudio definitivo hecho por algún organismo -público o privado- que indique, con mayor precisión, la situación actual de este rubro.

El último diagnóstico integral del sector artesanal lo desarrolló el Ministerio de Industria, Turismo, Integración y Negociaciones Comerciales Internacionales (Mitinci), en el año 1978; es necesario entonces revisar nuevamente su entorno y administración con la finalidad de replantear su futuro. Sabemos, además, que por disposición de la Dirección de Artesanía del Mitinci, se ha programado la ejecución del diagnóstico del sector, así como han iniciado coordinaciones para un trabajo conjunto con la Universidad de Lima, a partir del presente proyecto de investigación.

Hay instituciones que actualmente brindan apoyo al sector artesanal y también apuestan por su desarrollo, como la Asociación de Exportadores (Adex)-Convenio MSP USAID, Mitinci, Prompex (Comisión para la Promoción de Exportaciones) y diferentes organizaciones no gubernamentales (ONG). 
La Universidad de Lima, a través de la Dirección Universitaria Coordinadora de Investigación Científica (Ducic), ha estimado conveniente estimular y auspiciar proyectos que contribuyan a impulsar la modernización del sector artesanal como una alternativa para contribuir al desarrollo económico del país.

Planteamiento del problema desde el punto de vista de la ingeniería industrial

La artesanía, como actividad productiva en nuestro país, posee características definidas, desarrollándose en forma espontánea y empírica, con bajos montos de inversión, uso de tecnologías tradicionales, predominio de habilidad manual, basada en la capacidad creativa del artesano y con una limitada división del trabajo. Esta situación ubica al sector artesanal en desventaja en relación con otras actividades económicas y plantea un problema de urgente solución.

Desarrollar el sector artesanal implica demostrar que su producción posibilita la formación y acumulación de capital para que el artesano se enfrente sin desventaja a las nuevas condiciones del mercado, tanto para la adquisición de sus materias primas e insumos como para la venta de sus productos.

\section{Objetivos}

\section{Objetivos generales}

- Evaluar la situación técnica y administrativa actual de la actividad artesanal en el Perú.

- Plantear sugerencias para la adecuación de la tecnología artesanal a las exigencias de la economía moderna.

\section{Objetivos especificos}

- Participación de la Universidad de Lima, a través de la Facultad de Ingeniería Industrial, en el desarrollo y promoción de la actividad artesanal.

- Efectuar un diagnóstico del sector artesanal peruano, en el aspecto tecnológico y, complementariamente, en el comercial y social. 
- Promover la creación de los círculos de investigación y estudio en la Universidad de Lima, que incentivan el desarrollo del sector artesanal.

- Promover e impulsar el desarrollo de la artesanía peruana, como alternativa para el crecimiento de la economía nacional.

\section{Hipótesis}

- Dentro de una economía mundial moderna, la artesanía peruana tiene oportunidades para competir.

- El sector artesanal peruano posee un conjunto de debilidades técnicas y administrativas que le restan competencia en el mercado e impiden su desarrollo.

- Algunas líneas y procesos artesanales deben modernizarse con el fin de desarrollar algunos criterios de competencia, tales como diseño, volumen, precio y calidad.

- No existen políticas gubernamentales apropiadas que apoyen frontalmente el desarrollo del sector.

- El artesano, por mantener la existencia de su actividad artesanal, se resiste al cambio y a la modernidad, consecuentemente el desconocimiento de la tecnología moderna no le ha permitido usarla ni adaptarla a su tecnología propia.

- El sector artesanal posee escasos recursos económicos, lo que le impide invertir y elevar la calidad de vida de sus recursos humanos.

\section{Metodología}

El diagnóstico se basará fundamentalmente en los resultados de una encuesta denominada "Visita al taller", instrumento que será aplicado por personal capacitado.

Para realizar el estudio del diagnóstico artesanal se tendrá en cuenta el ámbito nacional, tomando como referencia la organización de las oficinas regionales de artesanía del Mitinci. En ese sentido, se utilizarán las líneas artesanales consideradas por aquella institución: textil, cerámica, platería, madera, cuero-peletería, fibra vegetal, bisutería, instrumentos musicales, piedra tallada, prendas de vestir, orfebrería, retablos, tejido a crochet, mates burilados y otros. 
Para la definición del tamaño de la muestra se considerará como universo el número de talleres formales registrados en el Mitinci y 100\% adicional sobre esta cantidad, con el fin de incluir los talleres informales; excepcionalmente, para los departamentos de Cusco, Junín, Puno y Lima se adicionará un incremento de $150 \%$, debido a la proporción de talleres formales e informales existentes en la actualidad.

La fórmula para el tamaño de muestra es:

$$
\mathrm{n}=\frac{4 P Q N}{\mathrm{e}^{2}(\mathrm{~N}-1)+4 P Q}
$$

donde:

$$
\mathrm{n}=\text { tamaño de la muestra }
$$

$\mathrm{P}=$ probabilidad que se realice

$\mathrm{Q}=$ probabilidad que no se realice

$\mathrm{N}=$ universo

$\mathrm{e}=$ error

El cuadro $\mathrm{N}^{\circ} 1$ contiene la cantidad de talleres artesanales a encuestar por departamento. 


\section{Cuadro $\mathrm{N}^{\circ} 1$ \\ Determinación del tamaño de la muestra de talleres artesanales por departamento}

\begin{tabular}{|c|c|c|c|c|c|c|}
\hline $\mathbf{N}^{\circ}$ & Departamento & $\begin{array}{c}\mathrm{N}^{0} \text { de talle- } \\
\text { res regis- } \\
\text { trados }\end{array}$ & $\begin{array}{c}\% \text { respecto } \\
\text { al total }\end{array}$ & $\begin{array}{c}\text { Estimado } \\
\text { total de } \\
\text { talleres }\left(^{*}\right)\end{array}$ & $\%$ real & $\begin{array}{c}\text { Tamaño de } \\
\text { muestra }\end{array}$ \\
\hline 01 & Amazonas & 11 & 0,05 & 22 & 0,04 & 01 \\
\hline 02 & Ancash & 613 & 2,60 & 1.226 & 2,04 & 10 \\
\hline 03 & Apurímac & 130 & 0,60 & 260 & 0,43 & 02 \\
\hline 04 & Arequipa & 2.120 & 9,09 & 4.240 & 7,07 & 35 \\
\hline 05 & Ayacucho & 1.711 & 7,34 & 5.133 & 8,56 & 43 \\
\hline 06 & Cajamarca & 979 & 2,91 & 1.944 & 3,24 & 16 \\
\hline 07 & Cerro de Pasco & 670 & 2,87 & 1.340 & 2,23 & 11 \\
\hline 08 & Cusco & 3.895 & 16,71 & 11.686 & 19,48 & 97 \\
\hline 09 & Huancavelica & 236 & 1,01 & 472 & 0,79 & 04 \\
\hline 10 & Huánuco & 228 & 0,98 & 456 & 0,76 & 04 \\
\hline 11 & Ica & 185 & 0,79 & 370 & 0.62 & 03 \\
\hline 12 & Junín & 2.478 & 10,63 & 7.734 & 12,39 & 62 \\
\hline 13 & La Libertad & 516 & 2,21 & 1.024 & 1,71 & 09 \\
\hline 14 & Lambayeque & 618 & 2,65 & 1.236 & 2,06 & 10 \\
\hline 15 & Loreto & 785 & 3,37 & 1.570 & 2,62 & 13 \\
\hline 16 & Madre de Dios & 35 & 0,15 & 70 & 0,12 & 01 \\
\hline 17 & Moquegua & 850 & 3,65 & 1.700 & 2,83 & 14 \\
\hline 18 & Piura & 932 & 4,00 & 1.864 & 3,11 & 16 \\
\hline 19 & Puno & 2.865 & 12,29 & 8.595 & 14,33 & 72 \\
\hline 20 & San Martín & 188 & 0,81 & 376 & 0,63 & 03 \\
\hline 21 & Tacna & 11 & 0,05 & 22 & 0,04 & 01 \\
\hline 22 & Tumbes & 83 & 0,36 & 166 & 0,28 & 01 \\
\hline 23 & Ucayali & 721 & 3,09 & 1.442 & 2,40 & 11 \\
\hline \multirow[t]{2}{*}{24} & Lima & 2.452 & 10,52 & 7.356 & 12,26 & 61 \\
\hline & Total & 23.312 & 100 & 60.004 & 100 & 500 \\
\hline
\end{tabular}

${ }^{*}$ ) Total de artesanos formales e informales.

Fuente: Adex, Mitinci, Prompex.

A la fecha se tiene concluida la aplicación de la encuesta en la muestra correspondiente a la ciudad de Lima. Complementariamente se utilizará información especializada sobre la participación del sector artesanal en la economía nacional, pronósticos de ventas en el mercado internacional por línea artesanal, diagnósticos anteriores desarrollados en la presente década, etc.; las fuentes de información complementarias serán tomadas de instituciones relacionadas con el sector en estudio: Adex, Mitinci, Prompex y organizaciones no gubernamentales. 


\section{Evolución de la artesanía en el Perú}

La artesanía en el Perú se remonta a miles de años atrás. Las manifestaciones artísticas de los antiguos peruanos estuvieron muy ligadas al sentimiento religioso. Las principales culturas que se desarrollaron en el Perú preincaico elaboraron diferentes tipos de actividades, como la metalurgia, textilería, arquitectura, música, mates burilados, etc., hechas con habilidades y técnicas sorprendentes. En la época del incanato se introdujo el predominio del culto al Sol.

La introducción de nuevas formas artísticas, traídas de Europa en el siglo XV, estuvo muy ligada al proceso de evangelización y transcurrió durante el tránsito de las expresiones antiguas a las nuevas. Durante la época colonial se produjeron cambios técnicos importantes, entre los cuales posiblemente el más notable fue la introducción del telar de pedal.

En el siglo XVIII, tras las rebeliones indígenas y sus consecuentes represiones, se produjo un fenómeno cultural muy complejo, que tuvo como característica el abandono de las formas artísticas quechuas y la adaptación de las europeas.

En el siglo XIX, luego de finalizado el Virreinato y la llegada de la era republicana, que trajo como consecuencia la desaparición de la aristocracia, los objetos pertenecientes a este sector social fueron sustituidos por otros más cercanos al contexto campesino y trabajados con técnicas menos elaboradas.

Ya en el siglo XX, durante el año 1945, se crearon los primeros talleres artesanales organizados y financiados por el Ministerio de Educación, con el fin de alentar el aprendizaje y las técnicas artesanales tradicionales. En 1960 se realizó la primera feria artesanal en el Parque Cánepa, en el distrito limeño de La Victoria. Estos talleres y ferias despertaron un cierto interés de parte de las nuevas generaciones por aprender artes tradicionales, así como también generaron una demanda urbana por estos objetos.

En 1970 se produjo una transformación en el campo del arte popular, debido a que la Junta Militar fomentó la artesanía tradicional, mediante la organización de festivales y ferias, que tuvieron como resultado el acercamiento del poblador urbano hacia lo andino. El programa Alianza para el Progreso, del gobierno de Estados Unidos, también apoyó la artesanía peruana, con la idea de promover la producción masiva y venderla en el 
mercado norteamericano, formándose las primeras compañías comercializadoras y exportadoras de este rubro.

Actualmente, la artesanía peruana se encuentra en tal situación que para ganar rápidamente competitividad se deben analizar factores como la reducción de costos y el aumento de la producción. Ésta comprende la fabricación de piezas de arte popular tradicional hechas en serie, como también de grupos de objetos creados en los últimos años para ser vendidos como elemento decorativo o funcional.

Es conveniente destacar la participación de diversas instituciones gubernamentales y privadas encaminadas al desarrollo del sector artesanal, como el convenio Adex-Usaid, el cual ha realizado una serie de proyectos con el propósito de mejorar las técnicas utilizadas, las materias primas, los productos finales, los diseños y la organización de talleres para que de esta manera la producción de esta actividad económica pueda competir con un mercado internacional y se pueda elevar la calidad de vida de los artesanos.

El Mitinci ha creado centros artesanales pilotos (actualmente en reactivación) con la finalidad de preparar la producción artesanal para la exportación. Asimismo, se planteó la necesidad de brindar capacitación y realizar innovaciones tecnológicas para agilizar los procesos.

\section{Principales líneas artesanales}

El Mitinci ha clasificado la producción artesanal en líneas tradicionales y líneas contemporáneas, agrupándolas sobre la base de criterios técnicos asociados a la forma de producción, materias primas y nivel de modernización.

\section{Lineas tradicionales}

\section{- $\quad$ Mates burilados}

Son productos artesanales trabajados en la superficie de la calabaza utilizando un buril, para realizar diversos diseños, como escenas de la vida cotidiana. El carácter de uso del producto será determinado por el artesano, ya que pueden considerarse utilitarios (joyeros) o decorativos.

Los principales lugares donde se producen estos mates son: Cochas (Huancayo), Chincha (Ica), Catacaos (Piura). 
- Cerámica

Hay diferentes tipos de cerámica, dependiendo del lugar de producción, debido a la diversidad étnica de la población peruana. La materia prima para elaborar los productos cerámicos es la arcilla roja de Ayacucho, Cusco, Chulucanas; y la arcilla blanca, proveniente de Ancash y Pucallpa.

Los principales lugares de producción de la cerámica son Quinua (Ayacucho), Pisac (Cusco), Pucará (Puno), Tarica (Ancash), Simbilá y Chulucanas (Piura), Yarinacocha (Ucayali).

- Textilería

Las fibras mayormente utilizadas son el algodón, pelo de camélidos y lana de ovino. La tecnología utilizada para la elaboración de los diferentes productos se basa principalmente en telares de mano, telares de cintura y en algunos casos telares mecánicos.

Los productos elaborados en esta línea son principalmente tapices, frazadas, alfombras, alforjas, cojines y bayetas.

Las zonas de mayor producción son: San Pedro de Cajas (Junín), el barrio de Santa Ana (Ayacucho), Hualhuas (Junín), Chota (Cajamarca), Huancané (Puno).

\section{- Imaginería}

Se originó a partir de la religiosidad colonial, y se realiza principalmente en los centros urbanos. Entre estos productos destacan los retablos, niños manuelitos y santos de cuello largo. La materia prima utilizada varía de acuerdo con el producto que se quiera elaborar.

Se producen principalmente en Ayacucho, Lima y el barrio de San Blas, en Cusco.

\section{- Tallado en piedra}

Se realizan en piedra de Huamanga (Ayacucho), marmolina (Cajamarca), caliza (Cusco) y sillar (Arequipa), elaborándose nacimientos, ceniceros, adornos con diseños incaicos y otros.

Los departamentos que destacan en este tipo de artesanía son Ayacucho, Cajamarca, Cusco y Arequipa. 


\section{- Platería}

En esta línea de artículos elaborados en plata están incluidas las categorías de orfebrería y filigrana.

Se produce principalmente en Lima, Cusco y San Jerónimo, en Junín.

\section{Contemporánea}

\section{- $\quad$ Tejido de punto}

En esta línea se ubican productos como chompas, cárdigans y otros accesorios de vestir, los cuales pueden estar fabricados basándose en fibra de alpaca, ovino o algodón.

Se produce sobre todo en Puno, Lima, Cusco, Junín, Huancavelica, Ica y Piura.

\section{- Fibras vegetales}

En esta línea se fabrican sombreros de paja, cestas, muebles elaborados de sauce, carteras, canastas, embarcaciones y hamacas.

Los principales centros de producción son Celendín, Jesús (Cajamarca), Monsefú (Lambayeque), Huacho (Lima), Chincha (Ica); Iquitos.

\section{- Madera}

Se fabrican muebles, candelabros y lámparas, entre otros; utlizándose como principal materia prima la caoba procedente de la selva peruana.

Estos productos se elaboran principalmente en Cusco, Lima, La Libertad y Apurímac.

\section{- Peletería}

Es el trabajo realizado en piel, principalmente de alpaca. Entre los productos que se fabrican dentro de esta línea están abrigos, alfombras, tapices y colchas.

En Juliaca, Cusco y Lima se fabrican abrigos; en Huancayo y Juliaca, alfombras y tapices; y en Sicuani (Cusco), colchas. 
- Otras líneas modernas

Destacan: arpillería, cuero, joyería, bisutería, muñecas, instrumentos musicales, pintura popular, entre otros.

\section{Producción artesanal regional}

Es importante considerar que para una mejor estratificación y análisis, la investigación ha tenido que identificar la producción artesanal predominante en cada región del país.

El mapa (véase p. 104) detalla la ubicación de las principales regiones artesanales; además, en el cuadro $\mathrm{N}^{\circ} 2$ se listan las principales ferias artesanales realizadas anualmente. 


\section{Cuadro $\mathrm{N}^{\circ} 2$}

Ferias artesanales a nivel nacional 1998

\section{Nombre}

\section{Organizadores}

Lugar

Bagua

Chachapoyas

Lamud

Caraz

Huaraz

Huaraz

Huaraz

Chimbote

Huaraz

Chiquián-Bolognesi

Huaraz
Municipalidad Prov. de Bagua

Comité Fiestas Patronales

Comité de Mayordomo

Concejo Provincial de Huaylas y

Asociación de Artesanos

Dir. Reg. Ind. y Turismo Consejo

Prov. Huaraz, Asoc. de Artesanos

Dir. Reg. Ind. y Turismo, Org. de Artesanos

Dir. Reg. Agricultura, Inst.

Públicas y Priv.

Conc. del Santa, Cámara

Comercio, Dir. Reg. Ind. y Turismo

Conc. Prov. Huaraz, Dir. Reg. Ind.

y Tur., Asoc. Artesanos

Conc. Prov. Bolognesi, Org.

Artesanos

Dir. Reg. Ind. y Turismo, Org.

Artesanos

Conc. Prov. Huamanga, Dir. Reg.

y Turismo

Conc. Prov. de Huanta

Conc. Prov. de San Miguel-La Mar

La Mar

Conc. Prov. Huamanga

Huamanga

Huamanga

Dir. Reg. Ind. y Turismo, Conc.

Prov. Huamanga

Dir. Reg. Ind. y Turismo, Conc.

Prov. Huamanga 


\section{CAJAMARCA}

Carnaval de Cajamarca

Feria San Juan Bautista

Feria de Huamantanga

Semana Turística y Cultural de Cajamarca

Todos los Santos

Feria Navideña

TACNA

ExpoTacna 98

\section{TUMBES}

Semana Turística de Tumbes

Tumbes

Feria Regional de Integración
Cajamarca

Chota

Jaén

Cajamarca

Cajamarca

Cajamarca

Fund. "La Agronómica"
Conc. Prov. Cajamarca, D.S.R. Ind. y Tur. Cajamarca Conc. Prov. de Chota Conc. Prov. de Jaén Dir. Subreg. Industria y Turismo Municipalidad de Cajamarca Dir. Subreg. Industria y Turismo

AgroTacna S.A.

Dir. Subreg. Tumbes

Conc. Prov. y/o Subprefectura

Comité de Feria (Cofesu)

Comité Feria de Talara

Comité de Feria de Catacaos

Comité de Feria Piura (Cofepi)

Comité Feria de Limón (Cofeli)

Comité Feria de Piura (Cofepi)

\section{Fedara}

Asoc. Emp. Cerro Juli

Asoc. Emp. Cerro Juli

Cerro Juli

Expo Agro 98

Feria Internacional

Descentralizada

Arequipa

Senati

Docsa

Asoc. Emp. Cerro Juli

Navi-Feria

Cerro Juli

Cusco

Dir. Reg. Ind. y Turismo

Feria Artesanal "Inti Raymi” 98

Feria Artesanal Plazoleta San

Francisco

Cusco

Cusco

Feria de Santurantikuy 98

Puno

Feria de las Alacitas

Juliaca
Frap. - Inka

Comisión Multisectorial

Comisión Organizad. de Munic.Puno

Emp. Promotora de Espectáculos Feitup 


\section{SAN MARTÍN}

Feria Artesanal y Comercial de Tarapoto

Feria Artesanal Semana

Turística de Lamas

Feria Agropecuaria y Artesanal

Feria Agrop. Agroindustrial y Artesanal

\section{HUÁNUCO}

Feria Artesanal

Feria Industrial, Comercial y Artesanal

\section{ICA}

Festival Internacional de la Vendimia

Aniversario de Pisco: Festival Fiestas Patrias

Señor de Luren

Navidad iqueña

\section{JUNíN}

\section{Feria Artesanal-Carnaval} Marqueño

Feria Exposición-Venta Artesanal Semana SantaHuancayo

Feria Internacional de la

Pachamanca

Festival del Folklore y la Pachamanca

Feria Artesanal de los Mates Burilados por Semana Santa Feria Artesanal por el Festival Turístico de La Merced XIV Feria de Exposición Artesanal Agrop. Artesanal e Industrial Expo 98

\section{IQUITOS}

Feria Nacional Artesanal, Folclórica San Juan 98

\section{MADRE DE DIOS}

Feria Agrop., Agroindustrial y Artesanal de Madre de Dios

\section{Tarapoto}

Lamas

Tocache

Juanjuí-Tarapoto

Huánuco

Huánuco

Ica

Ica

Ica

Ica

Marco- Jauja

Huancayo

Huancayo

Yauris-Huancayo

Cochas Chico-Huancayo

La Merced-Chanchamayo

San Juan-Iquitos

Madre de Dios
Conc. Prov. San Martín

Conc. Prov. Lamas

Conc. Prov. Tocache, Comité

Organizador de Feria

Comité de Feria

Comité Gremial de Artesanos

Conc. Prov. de Huánuco

Dir. Subreg. Ind. y Turismo Ica, Asoc. Artesanos, Conc. Prov. Ica Dir. Subreg. Ind. y Turismo Ica

Dir. Subreg. Ind. y Turismo Ica Dir. Subreg. Ind. y Turismo Ica

Dir. Subreg. Ind. y Turismo Junín

Dir. Subreg. Ind. y Turismo Junín

Dir. Subreg. Ind. y Turismo Junín Industria y Turismo-Junín, Oficina Zonal-San Ramón Empresa privada

Dir. Reg. de Ind. y Turismo, Asoc. de Artesanos de lquitos

Dir. Subreg. Agricultura, Dir., Subreg. Ind y Turismo de Madre de Dios 


\section{PASCO}

$X$ Expo 98 Feria Agropecuaria,

Artesanal, Industrial y

Folkclórica

Rancas

XV Expo 98 Meseta del Bombón.

Feria Regional Agrop. y

Artesanal de Ninacaca

Ninacaca

Multisectorial, Dir. Subreg.

Pasco

Multisectorial, Dir. Subreg.

Pasco

\section{LA LIBERTAD}

II Expo Primaveral de

Artesanía $98 \quad$ Trujillo

Exposición de Textiles y

Confecciones

Trujillo

Liga Artes. y Obreros del Perú.

Dir. Reg. de Ind. y Turismo

Asoc. Peq. Ind. de Tejidos de La

Libertad, Liga de Artes. del Perú

\section{LAMBAYEQUE}

Festival del Limón

Fexticum

Olmos

Monsefú

Municipalidad de Olmos

Municipalidad de Monsefú y

Comisión Organizadora

Santísima Cruz del Chalpón Motupe

Municipalidad de Motupe y

Hermandad

Semana Turística de Chiclayo Chiclayo

Dir. Ind. y Tur. Lambayeque

Actividades institucionales para promover el desarrollo artesanal en el país

El actual gobierno, a través del Mitinci, viene propiciando diversos programas para apoyar la promoción del sector artesanal, a través de diversas actividades organizadas.

Desde 1994 se estableció el Convenio Adex-Usaid en el Perú, a través del cual se fomentan las tradiciones artísticas y el bienestar comunitario. Uno de los logros del convenio es la presentación exitosa de productos artesanales en la Feria Internacional Gift Show de Nueva York, y se han organizado conferencias sobre crédito financiero y diseño.

La Comisión para la Promoción de Exportaciones (Prompex) presta apoyo al sector a través de la organización de ferias y capacitación en gestión y comercialización. Según Prompex las empresas artesanales peruanas participarán en las ferias de Alemania, Japón, Canadá, Suiza y España.

Resulta también importante la contribución de instituciones menores que apoyan al sector, entre las cuales se encuentran los gremios artesanales que buscan ayudar técnica y económicamente a sus asociados, como la Asociación Tawaq. Asi- 
mismo destaca la labor desarrollada por algunas organizaciones no gubernamentales que ofrecen capacitación en las áreas comercial y social, como la Asociación Minka, que intenta conseguir un mejor nivel de vida para el artesano y sus familiares.

Proyección de la demanda internacional para las principales lineas artesanales peruanas

Utilizando información histórica proporcionada por Adex, se ha efectuado una proyección de la demanda para los años 1999 y 2000, en las principales líneas artesanales: textil, cerámica, orfebrería y peletería.

\section{Gráfico $\mathbf{N}^{\circ} 1$}

Porcentaje de demanda de exportación para 1999

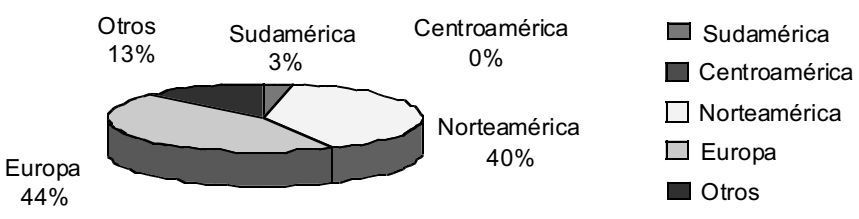

Gráfico No 2

Porcentaje de demanda de exportación para el 2000

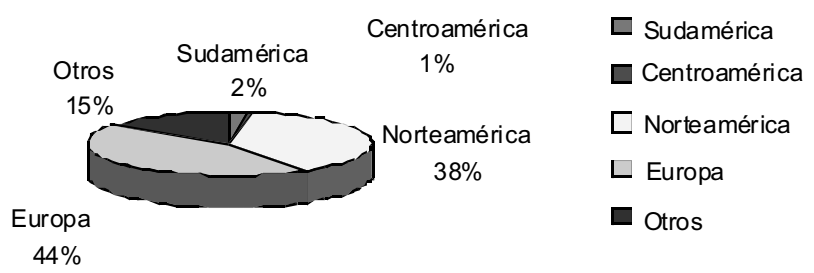




\section{Cuadro $\mathrm{N}^{\circ} 3$}

Demanda de la línea de cerámica

(Dólares FOB)

\begin{tabular}{lcccccc}
\hline & Sudamérica & Centroamérica & Norteamérica & Europa & Otros & Total \\
\hline 1994 & 1222015 & 460902 & 1535413 & 2152563 & 383346 & 5754239 \\
1995 & 2287547 & 653911 & 2285465 & 2249347 & 366756 & 7843025 \\
1996 & 2961372 & 919137 & 2690848 & 2240061 & 370033 & 9181451 \\
1997 & 3172790 & 1221947 & 3560691 & 2074147 & 324405 & 10353979 \\
1998 & 3038066 & 1544913 & 3970091 & 1829444 & 236389 & 10618903 \\
1999 & 3561033 & 2189425 & 4651877 & 1861826 & 235306 & 12499466 \\
2000 & 3750368 & 2968537 & 5266335 & 1787681 & 201679 & 13974600 \\
& & & & & \\
\hline
\end{tabular}

\section{Gráfico $N^{\circ} 3$}

\section{Tendencia de la demanda de la línea de cerámica}

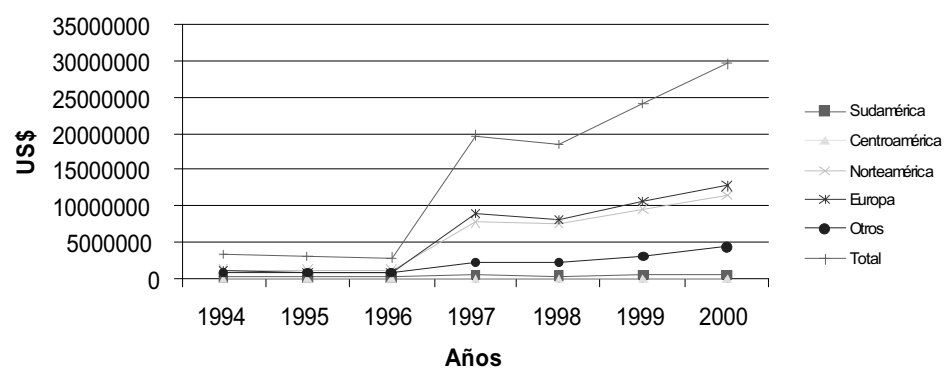




\section{Cuadro $\mathrm{N}^{\circ} 4$ \\ Demanda de la línea de peletería \\ (Dólares FOB)}

\begin{tabular}{lccccrc}
\hline Año & Sudamérica & Centroamérica & Norteamérica & Europa & Otros & TOTAL \\
\hline 1994 & 12448 & 16435 & 904257 & 108930 & 6598 & 1048667 \\
1995 & 16167 & 4516 & 897056 & 98128 & 8525 & 1024392 \\
1996 & 10138 & 21669 & 1087079 & 121849 & 21227 & 1261963 \\
1997 & 12839 & 56 & 1152424 & 150683 & 24836 & 1340838 \\
1998 & 19314 & 2580 & 1274990 & 127775 & 19968 & 1444628 \\
1999 & 17303 & 13478 & 1362211 & 150423 & 29219 & 1572635 \\
2000 & 18343 & 33 & 1461895 & 162107 & 33356 & 1675734 \\
\hline
\end{tabular}

\section{Gráfico $N^{\circ} 4$}

Tendencia de la demanda de la línea de peletería

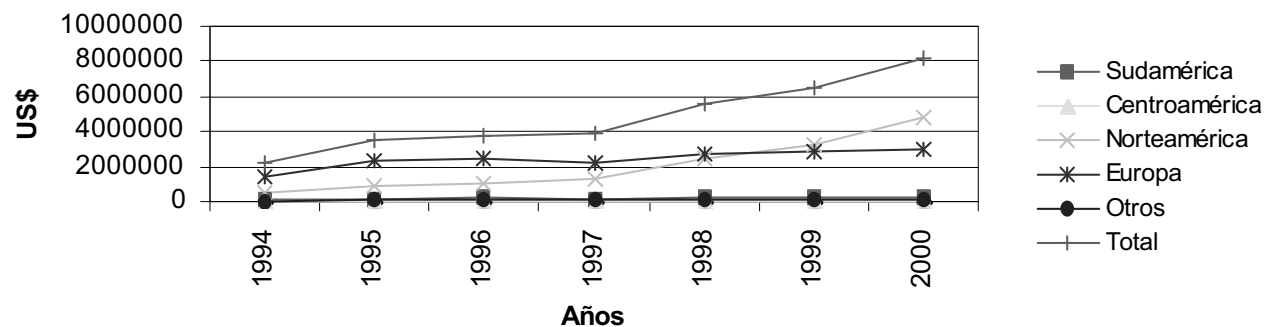




\section{Cuadro $\mathrm{N}^{\circ} 5$ \\ Demanda de la línea de orfebrería \\ (Dólares FOB)}

\begin{tabular}{lccccrc}
\hline AÑO & Sudamérica & \multicolumn{2}{c}{ Centroamérica Norteamérica } & Europa & Otros & Total \\
\hline 1994 & 153419 & 4275 & 476891 & 1464212 & 58128 & 2156925 \\
1995 & 173080 & 1453 & 880359 & 2353542 & 79719 & 3488153 \\
1996 & 207909 & 1608 & 981806 & 2418220 & 102309 & 3711853 \\
1997 & 187681 & 1896 & 1350177 & 2174737 & 148526 & 3863017 \\
1998 & 223728 & 1520 & 2501149 & 2672018 & 124279 & 5522693 \\
1999 & 223894 & 887 & 3283057 & 2823238 & 154491 & 6485567 \\
2000 & 231352 & 654 & 4773045 & 2963212 & 168247 & 8136510 \\
\hline
\end{tabular}

Gráfico $\mathbf{N}^{\circ} 5$

Tendencia de la demanda de la línea de orfebrería

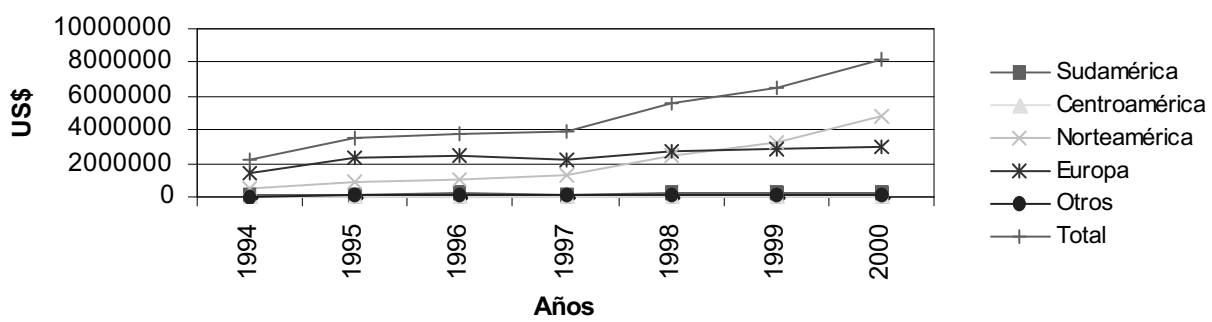

\section{Cuadro $\mathrm{N}^{\circ} 6$}

Demanda de la línea textil

(Dólares FOB)

\begin{tabular}{lrrrrrr}
\hline Año & Sudamérica & Centroamérica Norteamérica & Europa & \multicolumn{1}{c}{ Otros } & \multicolumn{1}{c}{ Total } \\
\hline 1994 & 216002 & 1315 & 1227506 & 1030341 & 806772 & 3281936 \\
1995 & 140843 & 574 & 1298903 & 862753 & 763737 & 3066810 \\
1996 & 105874 & 1913 & 1163153 & 893363 & 809318 & 2973621 \\
1997 & 711061 & 907 & 7772890 & 8963379 & 2352658 & 19800895 \\
1998 & 420589 & 7068 & 7647523 & 8119375 & 2245386 & 18439941 \\
1999 & 612692 & 5907 & 9616201 & 10657450 & 3143702 & 24035952 \\
2000 & 710631 & 7090 & 11547602 & 12885319 & 4317287 & 29467929 \\
\hline
\end{tabular}




\section{Gráfico $N^{\circ} 6$}

\section{Tendencia de la demanda de la línea textil}

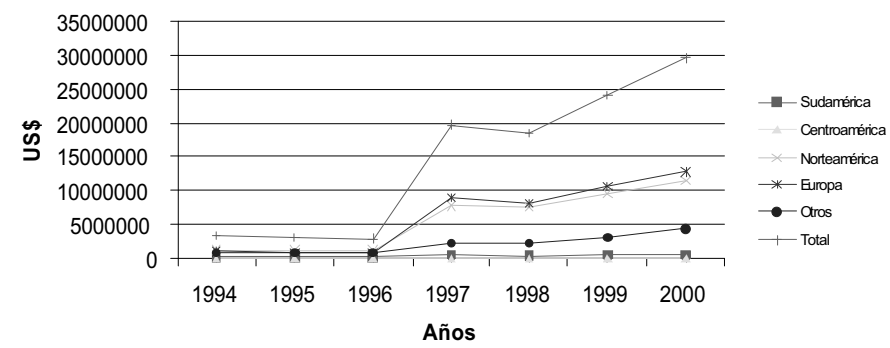

Como puede apreciarse, la artesanía peruana tiene una gran oportunidad de ventas en el mercado internacional, sin embargo, es necesario que los productos artesanales peruanos respondan a los requerimientos vigentes en el mercado internacional: bipolaridad social que busca combinar lo moderno y lo étnico, combinación de lo decorativo con lo utilitario, exigencia por la calidad y variedad sin dejar de lado el precio racional del producto. La tendencia puede alcanzar una mayor pendiente si cuidamos de mantener y fortalecer el posicionamiento actual. En ese sentido, el presente estudio pretende, a través del diagnóstico del sector, identificar las fortalezas y debilidades en las diferentes fases del proceso artesanal, con el fin de plantear sugerencias para su optimización y desarrollo. En un próximo artículo daremos a conocer los resultados y conclusiones más importantes obtenidas a partir de la aplicación de las encuestas utilizadas por el proyecto de investigación y a las cuales hace referencia este artículo. 


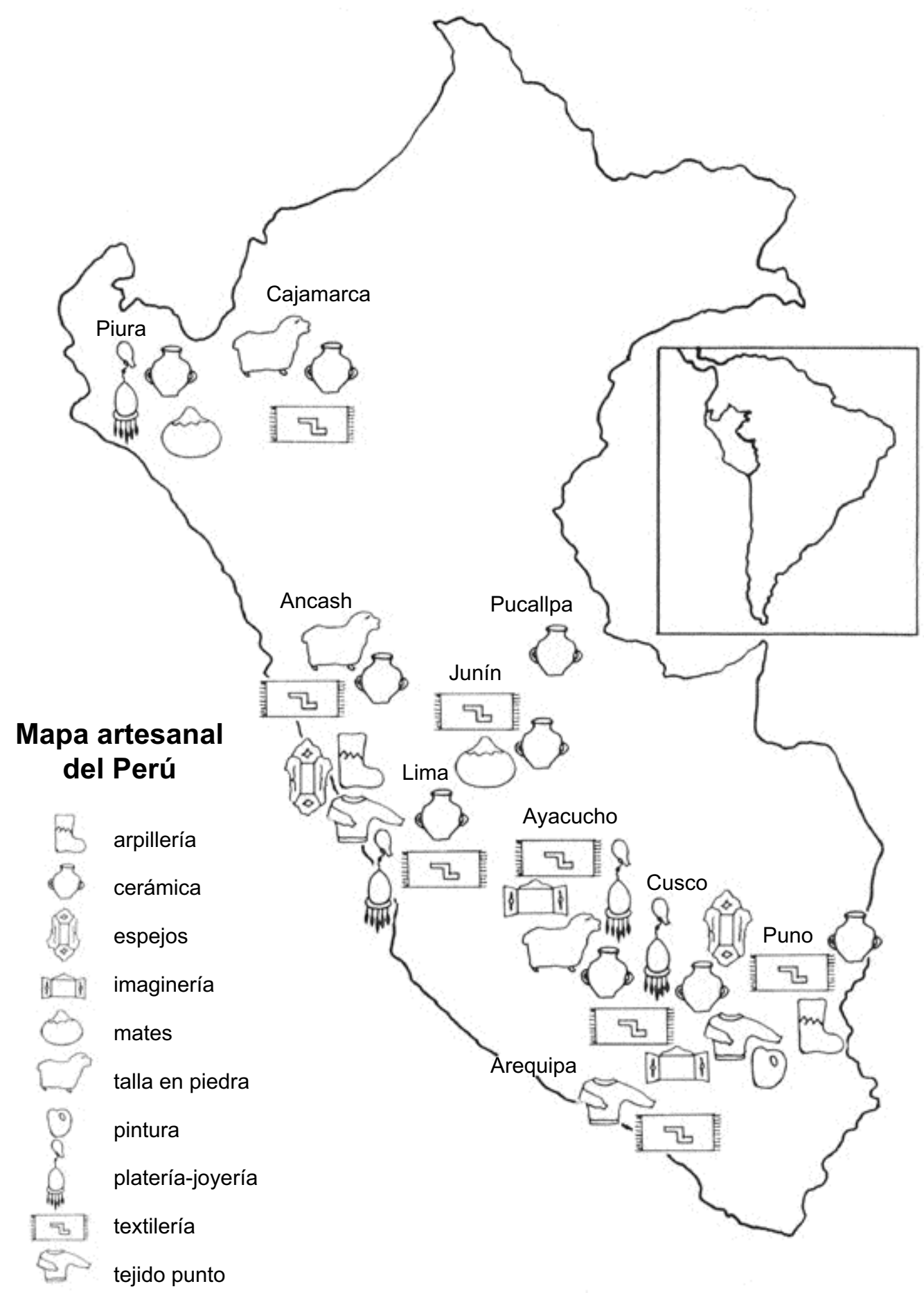




\section{Bibliografía}

Agencia Española de Cooperación Internacional

La artesanía iberoamericana en la Europa del 93. Perú: relación de productos de artesanos. Madrid: Ministerio de Industria, Comercio y Turismo, 1992.

Banco Industrial del Perú

Pequeña industria y artesanía, bibliografía. Lima: BIP, 1972.

- Pequeña empresa y artesanía; estudio bibliográfico. Lima: BIP, 1986.

Comunidad Iberoamericana de Artesanía

"Área de comercialización: análisis de mercados". Cuadernos de artesanía. Materiales didácticos para la formación de formadores del sector artesano de Iberoamérica: Formación a distancia. Madrid, 1997.

—. "Área de comercialización: distribución comercial". Cuadernos de artesanía. Materiales didácticos para la formación de formadores del sector artesano de Iberoamérica: Formación a distancia. Madrid, 1997.

Convenio Adex-Usaid

Boletín Informativo. Lima: Adex, 1997-1998 hasta junio 1999.

Delgado Candia, Sonia y René Clemente Camacho Merma

Artesanía y artesanos del Cusco, empresarios del futuro. Cusco: Centro de Estudios Regionales Andinos Bartolomé de las Casas, 1996.

De la Fuente, María del Carmen y otros

Artesanía peruana, orígenes y evolución. Arequipa: Allpa, 1990.

Fondo de Promoción de Exportaciones no Tradicionales Artesanías del Perú. Handicrafts. Lima: Fopex, 1989, IV.

“Artesanía: Nuevas estrategias de exportación”. Intercambio 111. Lima, febrero 1991. 
Plunket, James

"La artesanía: respuesta al desempleo en el Perú". Perú Exporta 214. Lima, junio 1994.

Rodríguez, Carlos

La artesanía peletera en alpaca; información básica del sector. Lima: Ideas, 1993.

Rojas, Julio (ed.)

Registro unificado. Códigos clasificatorios; comercio, industria, turismo, artesanía, transportes, salud. Lima: Roser, 1993.

Rojas Samanez, Gonzalo

“Artesanía peruana”. Supervisión 13. Lima, 1994.

Soto, Mary

"Esperando el boom”. Business 43. Lima, abril 1998.

Villanueva Cotrina, Víctor O.

Artesanía y cultura andina. 2a. edición. Cajamarca: Pratec, 1990. 